\title{
¿unisul
}

\section{REDUÇÃO DE DQO E TURBIDEZ EM EFLUENTE DE ABATEDOURO DE AVES TRATADO POR ELETROCOAGULAÇÃO}

\author{
Jaqueline Zanovelli Nalevaiko' \\ Everton Duim Rufato ${ }^{2}$ \\ Joel Gustavo Teleken ${ }^{3}$
}

\begin{abstract}
RESUMO
Mediante a alta produção de frangos de corte, os efluentes advindos dessa produção tornam-se um grande impasse ambiental por conta de sua elevada carga orgânica, características recalcitrantes e difícil assimilação no meio ambiente. Frente a isso, métodos alternativos como a eletrocoagulação são vistos como potencialmente eficientes no tratamento dos mesmos. O presente estudo tem por objetivo avaliar a possibilidade de utilização da eletrocoagulação no tratamento de efluente de um abatedouro de aves com alta carga orgânica, sem utilização de aditivos, por meio da análise de redução de Demanda Química de Oxigênio (DQO) e turbidez do efluente, variando o potencial hidrogeniônico $(\mathrm{pH})$ e o tempo reacional. As melhores condições encontradas foram o tempo de 60 minutos e $\mathrm{pH} 7$, obtendo $68,1 \%$ de redução de DQO e $78,3 \%$ de redução de turbidez. O tratamento sem adição de sais aditivos se mostrou viável para ser utilizado como tratamento secundário, principalmente por ser rápido.
\end{abstract}

Palavras-chaves: Tratamento de efluente; Agroindústria; Eletrólise.

1 Mestranda em Bioenergia, Universidade Federal do Paraná (UFPR). E-mail: jaqueline.nalevaiko@gmail.com

\footnotetext{
2 Professor do curso de Engenharia Civil, Faculdade de Iporã. E-mail: everton.rufato@hotmail.com

3 Professor do departamento de Engenharias e Exatas, Universidade Federal do Paraná (UFPR). Email: joel.teleken@ufpr.br
} 


\title{
DQO AND TURBITY REDUCTION IN POULTRY SLAUGHTERHOUSE EFFLUENT TREATED BY ELECTROCOAGULATION
}

\begin{abstract}
Due to the high production of broilers, the effluents from this production become a major environmental impasse due to its high organic load, recalcitrant characteristics and difficult assimilation into the environment. Therefore, alternative methods such as electrocoagulation are seen as potentially efficient in their treatment. This study aims to evaluate the possibility of using electrocoagulation in the treatment of effluent from a poultry slaughterhouse with a high organic load, without using additives, through the analysis of reduction of Chemical Oxygen Demand (COD) and turbidity of the effluent, varying the hydrogen potential $(\mathrm{pH})$ and the reaction time. The best conditions found were the time of 60 minutes and $\mathrm{pH} 7$, obtaining $68.1 \%$ of COD removal and $78.3 \%$ of turbidity reduction. The treatment without addition of additive salts proved viable to be used as secondary treatment, mainly because it is fast.
\end{abstract}

Keywords: Wastewater treatment; Agroindustry; Electrolysis.

\section{INTRODUÇÃO}

O crescimento econômico do Brasil nas últimas décadas está ligado ao crescimento do agronegócio, principalmente devido ao aumento do consumo interno e à expansão do mercado de exportação de proteína animal. A avicultura tem participação significativa nesse contexto, e de acordo com a Empresa Brasileira de Pesquisa Agropecuária - EMBRAPA (2019) o estado do Paraná é o maior produtor e exportador de frangos de corte do Brasil.

Tal aumento na expansão do mercado de carne de aves é acompanhado pelo desenvolvimento da tecnologia de abate e instalação de abatedouros de alta capacidade, acarretando em um aumento na quantidade de resíduos gerados na produção e processamento, e também em um aumento na geração de efluentes líquidos, o que é preocupante do ponto de vista ambiental, pois o mesmo apresenta alta carga orgânica e características recalcitrantes, tornando o tratamento mais difícil (MÓDENES et al., 2017; COMBATT et al., 2017; KRUG \& HERMES, 2019).

O efluente do processamento de aves, em geral, possui alta demanda química de oxigênio, elevado teor de sólidos, alta turbidez e cor opaca - devido à presença de restos de carne, vísceras, penas, ossos e gordura animal - além de apresentar pH levemente ácido (FERREIRA et al. 2018). 
GESTÃO \& SUSTENTABILIDADE AMBIENTAL

O tratamento dos efluentes ganha uma certa preocupação por parte da indústria de carnes devido a motivos como: restrições legais para a disposição de efluentes, custos de tratamento, consumidores ambientalmente conscientes, busca por alternativas menos impactantes e mais eficientes entre outros (COMBATT et al., 2017).

Os tratamentos têm como objetivo atender a legislação ambiental para o lançamento dos efluentes nos corpos hídricos, e em alguns casos fazer o reuso da água. Segundo An et al. (2017) essas condições de remoção podem ser alcançadas por diferentes tipos de tratamento, sendo que os tratamentos de efluentes mais comuns utilizam um agente coagulante e lagoas flutuantes, anaeróbicas e facultativas. Apesar da disponibilidade destes métodos eles apresentam algumas desvantagens como baixa eficiência, longo tempo de retenção e necessidade de grande área de tratamento, causando mau cheiro, além de não serem eficientes na remoção de substâncias que sejam nocivas aos microrganismos (LIMA e ALVES, 2018).

Algumas alternativas de tratamento que possam ser mais vantajosas vêm sendo estudadas. Yavuz e Ogutveren (2018), Abreu et al. (2019), Silva et al. (2020), Souza et al. (2016) destacam como alternativa ao tratamento convencional o processo de eletrocoagulação como uma tecnologia eficaz. O tratamento por eletrocoagulação consiste na passagem da corrente elétrica por um determinado efluente, desencadeando diversas reações: substituição iônica entre eletrólitos inorgânicos e sais orgânicos, oxidação de compostos, redução da concentração de matéria orgânica dissolvida e desestabilização de partículas coloidais.

A eletrocoagulação é influenciada por algumas variáveis físico-químicas, tais como: $\mathrm{pH}$ do efluente, corrente elétrica e presença de sais ou eletrólitos. De acordo com Moussa et al. (2017) a corrente elétrica inserida no eletrocoagulador é diretamente proporcional à remoção da demanda química de oxigênio (DQO), mas não é possível obter alta corrente se a condutividade da solução for baixa. De acordo com Hakizimana et al. (2017) a adição de qualquer composto para manter a corrente alta aumenta o custo do processo e a quantidade de sólidos solúveis no efluente tratado.

O processo de eletrocoagulação ocorre em três etapas que envolvem a formação de coagulantes por oxidação eletrolítica do eletrodo de sacrifício, a desestabilização da suspensão de contaminantes em partículas e a formação de 
flocos. A desestabilização das moléculas que estão no efluente é gerada por meio do diferencial de potencial elétrico aplicado aos eletrodos, assim as espécies catiônicas são geradas pelo ânodo, reagindo com os coloides da solução tratada e gerando a flotação do material (KRUG \& HERMES, 2019).

No processo de tratamento de efluentes, os íons são gerados no ânodo e reagem com os hidróxidos formados no cátodo (hidrólise da $\mathrm{H}_{2} \mathrm{O}$ ). Os hidróxidos de metais produzidos na reação reagem com os sólidos suspensos e coloides formando os precipitados (SRIDHAR et al., 2012). Segundo Thirugnanasambandham et al. (2014), o mecanismo de eletrocoagulação ocorre da seguinte maneira:

$$
\begin{gathered}
\text { No ânodo } \\
\mathrm{M} \rightarrow \mathrm{M}^{\mathrm{n}+}+\mathrm{ne}^{-} \\
\text {No cátodo } \\
\mathrm{nH}_{2} \mathrm{O}+\mathrm{ne}^{-} \rightarrow \mathrm{H}_{2}+\mathrm{nOH}^{-}
\end{gathered}
$$

A eletrocoagulação apresenta vantagens como pouco espaço físico necessário no processo por batelada, equipamentos de fácil operação, baixo tempo de tratamento e flocos formados mais estáveis, facilitando a remoção deles. Dentre as desvantagens do processo estão o alto consumo de eletricidade e a necessidade de substituição regular dos eletrodos. Essa substituição se deve à passivação do eletrodo, devido à formação de uma película de produto de corrosão (KUMAR et al., 2009).

Em tratamentos de efluentes de abatedouros de aves ainda pouco se utiliza tratamentos alternativos. O objetivo do presente estudo é verificar se o tratamento por eletrocoagulação é uma boa opção de tratamento para esse tipo de efluente, avaliando a mudança nas variáveis $\mathrm{DQO}$, turbidez e condutividade após o tratamento por eletrocoagulação sem o uso de sais ou aditivos. Pretende-se ainda verificar a melhor relação entre as variáveis tempo de eletrocoagulação e pH.

\section{MATERIAIS E MÉTODOS}

Para a realização deste estudo dividiu-se em diferentes etapas: coleta e caracterização do efluente, construção do eletrocoagulador de bancada, ensaios de tratamento por eletrocoagulação, processamento e análise de dados.

Foi realizada a coleta de 40 litros de efluente em um abatedouro de aves, após a linha de abate, local onde ocorre apenas o tratamento preliminar por meio de 


\section{¿UISUl Somima}

peneiramento para retirada de sólidos grosseiros. Tal efluente é composto por resíduos de carnes, penas, sangue, microrganismos patogênicos e outros resíduos orgânicos advindos do processo de abate de aves. Para armazenagem foram utilizados frascos âmbar de vidro, mantidos sob refrigeração a $4^{\circ} \mathrm{C}$ de acordo com a NBR 9898/87 (ABNT, 1987).

O efluente bruto foi homogeneizado e caracterizado quanto à Turbidez, DQO, condutividade e $\mathrm{pH}$. Os métodos utilizados na avaliação destes parâmetros são apresentados na Tabela 1. Os mesmos parâmetros foram analisados após o processo de eletrocoagulação.

Tabela 1 - Metodologia para determinação de Turbidez, DQO, Condutividade e pH.

\begin{tabular}{|c|c|c|c|}
\hline Parâmetro & Método & Análise & Equipamento \\
\hline Turbidez & Standard Methods & $\begin{array}{l}\text { Leitura } \\
\text { direta }\end{array}$ & Turbidímetro AP 2000 \\
\hline DQO & $\begin{array}{l}5220 \text { B do Standard } \\
\text { Methods }\end{array}$ & Colorimetria & DR2800 da HACH \\
\hline $\begin{array}{c}\text { Condutividad } \\
\mathrm{e}\end{array}$ & 2510 B Standard Methods & $\begin{array}{l}\text { Leitura } \\
\text { direta }\end{array}$ & $\begin{array}{c}\text { Condutivímetro } \\
\text { AP2000 }\end{array}$ \\
\hline $\mathrm{pH}$ & $\begin{array}{c}4500 \mathrm{H}+\mathrm{B} \text { Standard } \\
\text { Methods }\end{array}$ & $\begin{array}{l}\text { Leitura } \\
\text { direta }\end{array}$ & pHametro Mpa210 \\
\hline
\end{tabular}

Fonte: Adaptado de APHA (2012).

Para o processo de eletrocoagulação foi construído um eletrocoagulador de vidro em escala laboratorial com capacidade de 3 litros, tendo $20 \mathrm{~cm}$ de comprimento, $10 \mathrm{~cm}$ de largura, e $15 \mathrm{~cm}$ de altura. Dentro do recipiente foram instalados 4 eletrodos

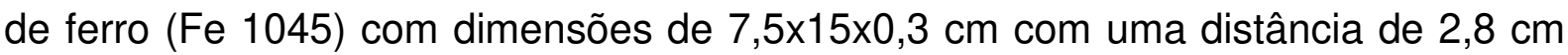
entre eles. Tais eletrodos foram presos a uma plataforma de madeira, e conectados a uma fonte de energia por um fio de cobre de $1,5 \mathrm{~mm}$, a fonte contínua com tensão 15 Volts (modelo Hahonik FTE 1310), que no início do processo fornecia uma corrente elétrica de 5,5 Ampere. Não foi feita a adição de sal ou outro tipo de material O pH inicial de 5,75 foi reajustado no início de cada um dos ensaios por meio da adição de Hidróxido de Sódio 0,1 M, para deslocá-lo para regiões menos ácidas, visto que os processos de eletrocoagulação são fortemente dependentes do $\mathrm{pH}$, pois íons liberados pelos eletrodos são difíceis de agregar-se sob condições ácidas. O único tratamento utilizado foi a eletrocoagulação (CHEN, 2004).

Empregou-se um planejamento fatorial $2^{3} \mathrm{com}$ três repetições no ponto central para condução da pesquisa utilizando-se como variáveis independentes o pH e tempo 


\section{GESTÃO \& SUSTENTABILIDADE}

AMBIENTAL

\section{$\checkmark$ UnISUl}

de eletrólise. Conforme planejamento obtiveram-se 11 unidades amostrais de forma aleatória. Utilizou-se 1,5 L de efluente no desenvolvimento de cada ensaio. Os valores e níveis utilizados são apresentados na Tabela 2.

Tabela 2 - Planejamento experimental.

\begin{tabular}{c|cc}
\hline № experimento & $\mathrm{pH}$ & teletrólise $(\mathrm{min})$ \\
\hline 1 & 6 & 45 \\
2 & 6 & 75 \\
3 & 6 & 60 \\
4 & 7 & 45 \\
5 & 7 & 75 \\
6 & 7 & 60 \\
7 & 8 & 45 \\
8 & 8 & 75 \\
9 & 8 & 60 \\
10 & 7 & 60 \\
11 & 7 & 60 \\
\hline
\end{tabular}

Fonte: Autores própria (2020).

Os valores utilizados para $\mathrm{pH}$ e tempo de eletrólise foram definidos com base em valores utilizados por outros autores, que encontraram os melhores resultados no processo de eletrocoagulação nas condições de pH entre 6 e 8 , e tempos entre $45 \mathrm{e}$ 75 minutos (BORBA et al., 2010; SANTOS et al., 2018). Todos os experimentos foram realizados em duplicata e para análise estatísticas dos dados utilizou-se o software Statitisca 8.0.

\section{RESULTADOS E DISCUSSÕES}

A caracterização do efluente bruto coletado após o tratamento primário do abatedouro de aves é apresentada na Tabela 3.

Tabela 3 - Caracterização do efluente bruto.

\begin{tabular}{c|c}
\hline Turbidez (NTU) & 453 \\
$\mathrm{pH}$ & 5,75 \\
$\mathrm{DQO}(\mathrm{mg} / \mathrm{L})$ & 5455 \\
Condutividade $\left(\mu \mathrm{s} / \mathrm{cm}^{2}\right)$ & 864 \\
\hline
\end{tabular}

Fonte: Autores (2020). 


\section{GESTÃO \& SUSTENTABILIDADE \\ AMBIENTAL}

\section{¿Unisul}

Após os processos de eletrocoagulação foram realizadas novas análises nas amostras, sendo os resultados obtidos apresentados na Tabela 4.

Tabela 4 - Caracterização do efluente após a eletrocoagulação.

\begin{tabular}{c|c|c|c|c|c|c}
\hline № & $\begin{array}{c}\text { Turbidez } \\
\text { (NTU) }\end{array}$ & $\begin{array}{c}\text { Remoção de } \\
\text { Turbidez }(\%)\end{array}$ & $\begin{array}{c}\text { DQO } \\
(\mathrm{mg} / \mathrm{L})\end{array}$ & $\begin{array}{c}\text { Remoção de } \\
\text { DQO }(\%)\end{array}$ & $\begin{array}{c}\text { Condutividade } \\
\left(\mu \mathrm{s} / \mathrm{cm}^{2}\right)\end{array}$ & $\begin{array}{c}\text { Variação de } \\
\text { Condutividade } \\
(\%)\end{array}$ \\
\hline 1 & 165 & 63,58 & 2731 & 49,94 & 1046 & 21,06 \\
2 & 151 & 66,67 & 2250 & 58,75 & 1222 & 41,44 \\
3 & 133 & 70,64 & 2019 & 62,99 & 1109 & 28,46 \\
4 & 130 & 71,30 & 2330 & 57,29 & 1070 & 23,84 \\
5 & 118 & 73,95 & 1922 & 64,77 & 1241 & 43,63 \\
6 & 102 & 77,48 & 1699 & 68,85 & 1129 & 30,67 \\
7 & 98 & 78,37 & 1755 & 67,83 & 1252 & 44,91 \\
8 & 100 & 77,92 & 1727 & 68,34 & 1191 & 37,85 \\
9 & 158 & 65,12 & 2555 & 64,80 & 1092 & 26,39 \\
10 & 140 & 69,09 & 2228 & 59,16 & 1262 & 46,06 \\
11 & 120 & 73,51 & 1976 & 63,78 & 1150 & 33,10 \\
\hline
\end{tabular}

Fonte: Autores (2020).

Na Figura 1 é apresentado o gráfico de Pareto para os resultados de remoção de turbidez após o processo de eletrocoagulação. Observa-se que o tempo quadrático, tempo linear, $\mathrm{pH}$ quadrático e $\mathrm{pH}$ linear tiveram influência significativa sobre o processo de eletrocoagulação no que se refere a redução da turbidez. 


\section{GESTÃO \& SUSTENTABILIDADE} AMBIENTAL

\section{$\checkmark$ UnISUl}

Figura 1 - Gráfico de Pareto: Análise de remoção de turbidez.

Redução de Turbidez (\%)

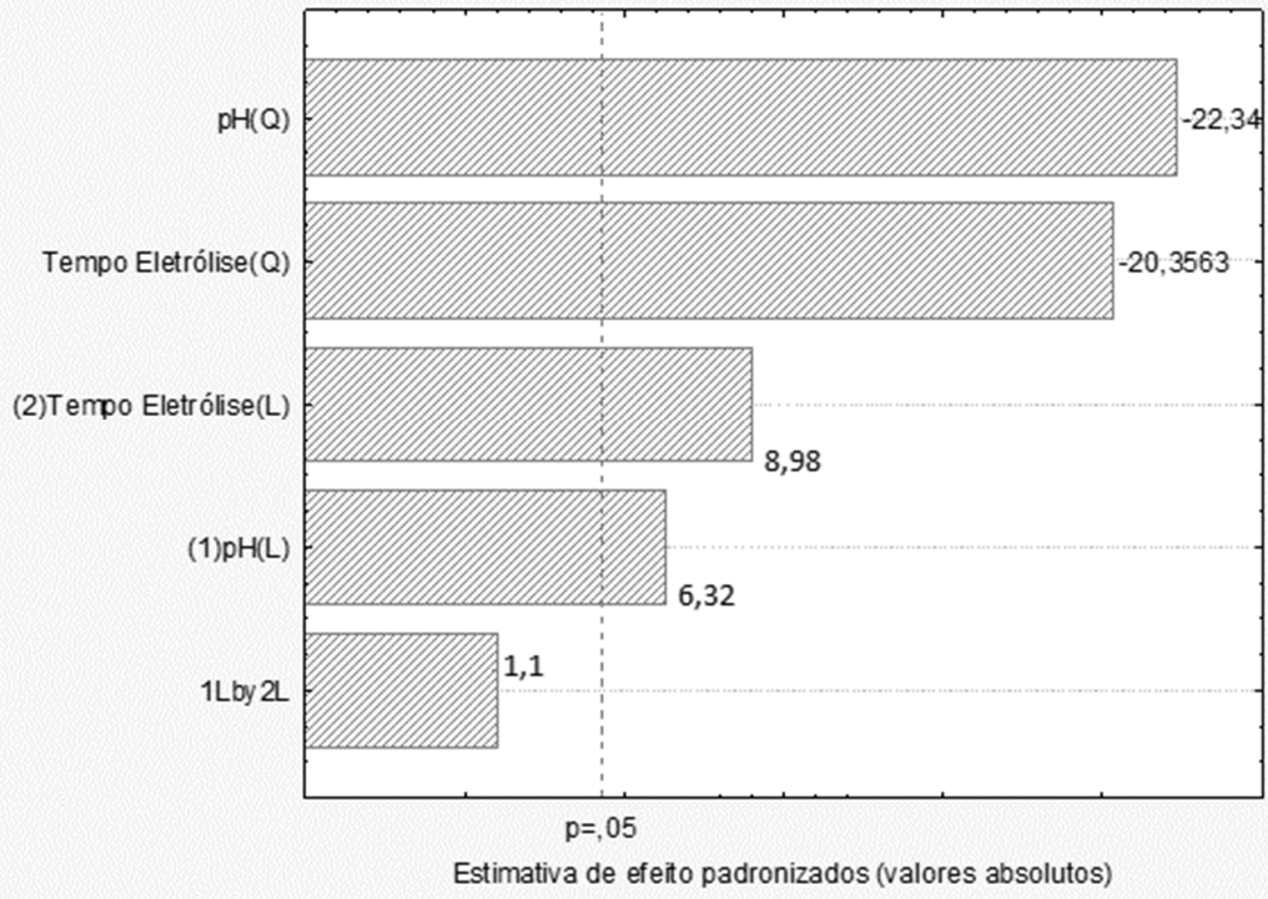

Fonte: Autores (2020).

A Tabela 5 traz o resultado da análise de variância ANOVA para a remoção da turbidez. A partir dos resultados apresentados pela ANOVA observa-se que o modelo gerado a partir dos dados experimentais é válido para este intervalo de $\mathrm{pH}$ e tempo de eletrólise testados.

Tabela 5 - Resumo ANOVA: Análise da turbidez.

\begin{tabular}{c|c|c|c}
\hline & $\mathrm{F}$ calculado & $\mathrm{p}$-valor & Efeitos \\
\hline $\mathrm{pH}$ (Linear) & 40,0417 & 0,024076 & 2,2811 \\
$\mathrm{pH}$ (quadrático) & 499,3373 & 0,001997 & $-12,3969$ \\
Tempo (linear) & 80,6667 & 0,012171 & 3,2377 \\
Tempo (quadrático) & 414,3789 & 0,002405 & $-11,2931$ \\
\hline \multicolumn{2}{|c}{$\mathrm{Z}=330,46+87,92 \mathrm{x}-6,20 \mathrm{x}^{2}+3,12 \mathrm{y}-0,03 \mathrm{y}^{2}$} \\
\hline
\end{tabular}

Onde: $\mathrm{Z}=$ Redução da Turbidez (\%); $\mathrm{x}=\mathrm{pH} ; \mathrm{y}=$ tempo de eletrólise; $\mathrm{p}$-valor inferiores $\mathrm{a}$ 0,05 indicam que o modelo explica a variação na resposta com significância estatística ao nível de $95 \%$ de probabilidade.

Fonte: Autores (2020).

O gráfico de superfície resposta gerado pelo comportamento da redução da turbidez em função do pH e do tempo de eletrólise dentro dos limites analisados é apresentado na Figura 2. 


\section{GESTÃO \& SUSTENTABILIDADE} AMBIENTAL

\section{¿UNISUl}

Figura 2 - Superfície resposta para análise de remoção de turbidez (\%).

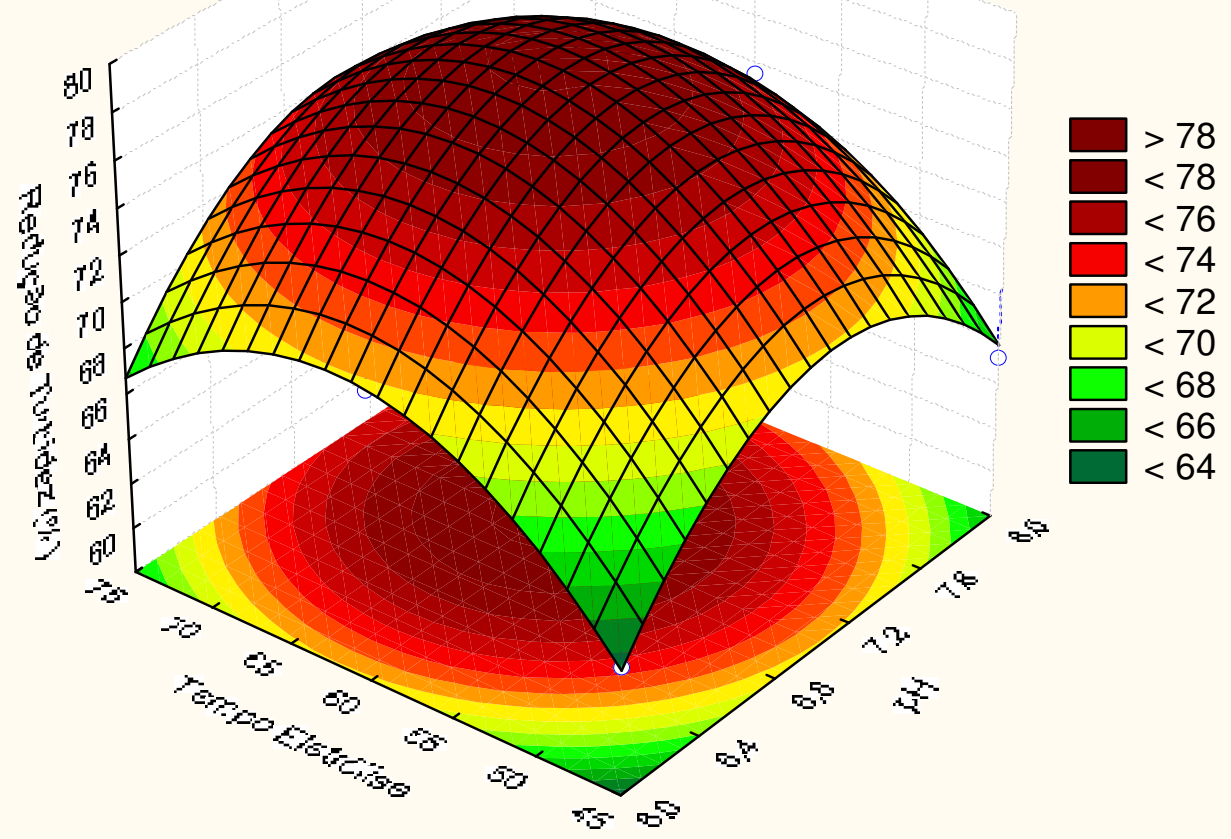

Fonte: Autores (2020).

A maior remoção de turbidez obtida foi de $78,3 \%$, no experimento 7 , onde o valor encontrado é de 98 NTU. O percentual de remoção da turbidez diminui com elevados tempos de reação devido ao decréscimo da condutividade no processo. Quando se utilizam processos contínuos, uma medida utilizada para o controle dessa condição é a adição de sais no meio.

Verificou-se que a redução da turbidez apresenta melhores resultados com valores de $\mathrm{pH}$ próximos a 7,2 e tempo de eletrólise de aproximadamente $60 \mathrm{~min}$, valores muito próximos ao ponto central do planejamento.

Em estudo semelhante Módenes et al. (2017) obtiveram valores de aproximadamente $99 \%$ de redução de turbidez para efluentes de abatedouros de aves, utilizando tempo de 60 minutos e corrente de $3 \mathrm{~A}$, porém utilizando compostos clorados inorgânicos e orgânicos para controle da condutividade.

Na Figura 3 é apresentado o Gráfico de Pareto para a análise de redução de DQO - parâmetro utilizado para medir a quantidade de oxigênio necessário para oxidar os compostos orgânicos presentes no efluente (ROSHINI et al., 2017; KUMAR et al., 2018). 


\section{GESTÃO \& SUSTENTABILIDADE}

AMBIENTAL

\section{¿Unisul sim}

Figura 3 - Gráfico de Pareto: Análise de remoção de DQO.

Redução DQO (\%)

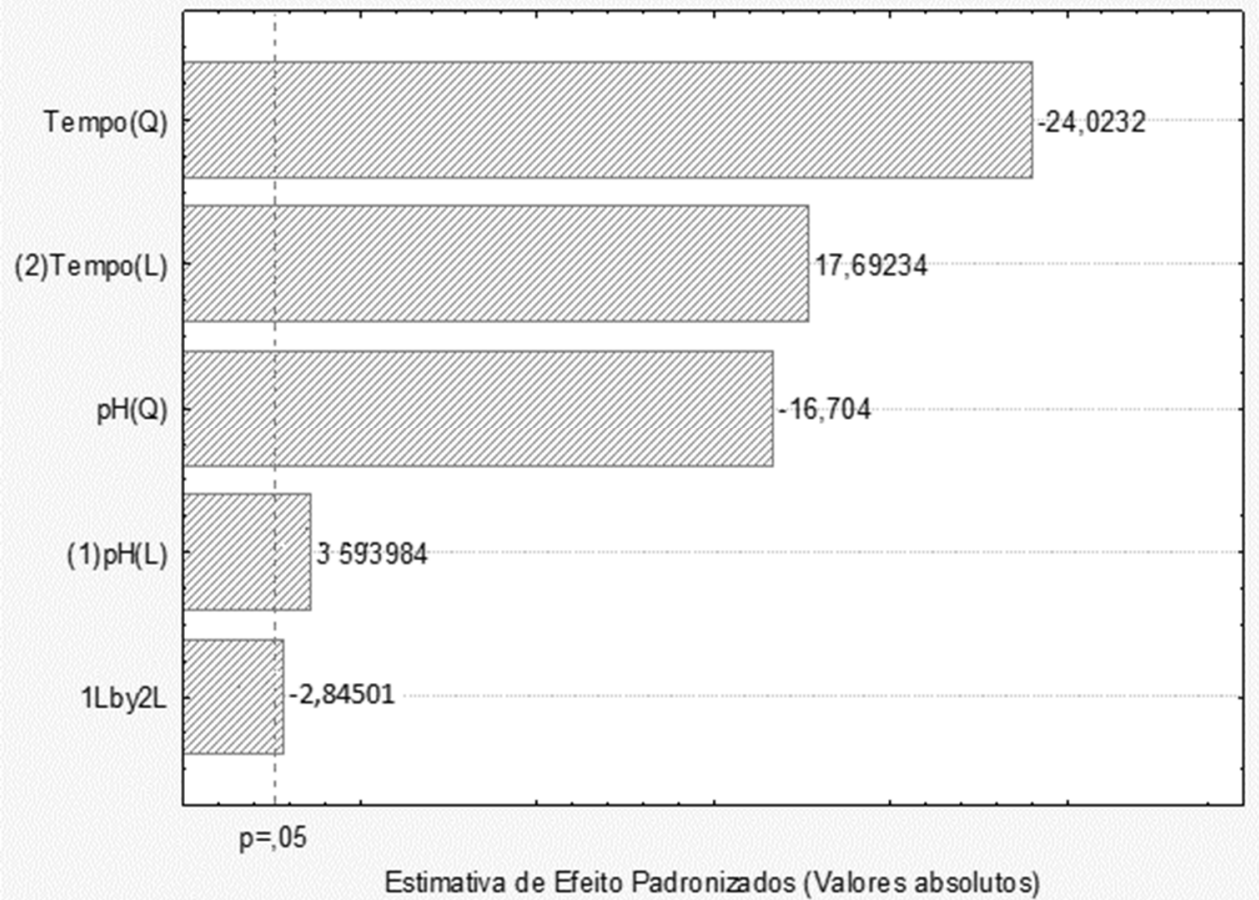

Fonte: Autores (2020).

Conforme apresentado, os termos de tempo quadrático, tempo linear, pH linear, $\mathrm{pH}$ quadrático, assim como a interação linear entre $\mathrm{pH}$ e tempo apresentaram influência significativa para redução da DQO pelo processo de eletrocoagulação.

A Tabela 6 apresenta a análise de variância ANOVA para análise de remoção de DQO, sendo que o modelo gerado a partir dos dados experimentais é válido para os intervalos de $\mathrm{pH}$ e tempo de eletrólise testados.

Tabela 6 - Resumo ANOVA: Análise de DQO.

\begin{tabular}{c|c|c|c}
\hline & F calculado & p-valor & Efeitos \\
\hline pH (Linear) & 12,9167 & 0,015642 & 1,5032 \\
pH (quadrático) & 279,0235 & 0,000014 & $-10,7521$ \\
Tempo (linear) & 313,0188 & 0,000011 & 7,3999 \\
Tempo (quadrático) & 577,1158 & 0,000002 & $-15,4634$ \\
Interação & 8,0941 & 0,036034 & $-1,4574$ \\
\hline
\end{tabular}

Modelo Matemático $\quad Z=-359,09+78,93 x-5,38 x^{2}+4,71 y-0,03 y^{2}-0,05 x y$

Onde: $Z=$ Redução da DQO (\%); $x=\mathrm{pH} ; y=$ tempo de eletrólise; $p$-valor inferiores a 0,05 indicam que o modelo explica a variação na resposta com significância estatística ao nível de $95 \%$ de probabilidade.

Fonte: Autores (2020). 


\section{GESTÃO \& SUSTENTABILIDADE AMBIENTAL}

\section{¿unisul sines}

Para DQO a maior redução encontrada foi de $68,8 \%$, no experimento 6 com DQO final de $1699 \mathrm{mg} / \mathrm{L}$. De acordo com o Instituto Ambiental do Paraná (IAT), para - lançamento de efluentes líquidos de empreendimentos de avicultura em corpos hídricos a DQO aceita é de até $150 \mathrm{mg} / \mathrm{L}$, sendo então apenas o tratamento por eletrocoagulação proposto insuficiente para atender a legislação (IAP, 2020).Na Figura 4 é apresentado comportamento da redução da DQO em função do pH e tempo de eletrólise para os limites estudados.

Figura 4 - Superfície resposta para análise de redução de DQO (\%).

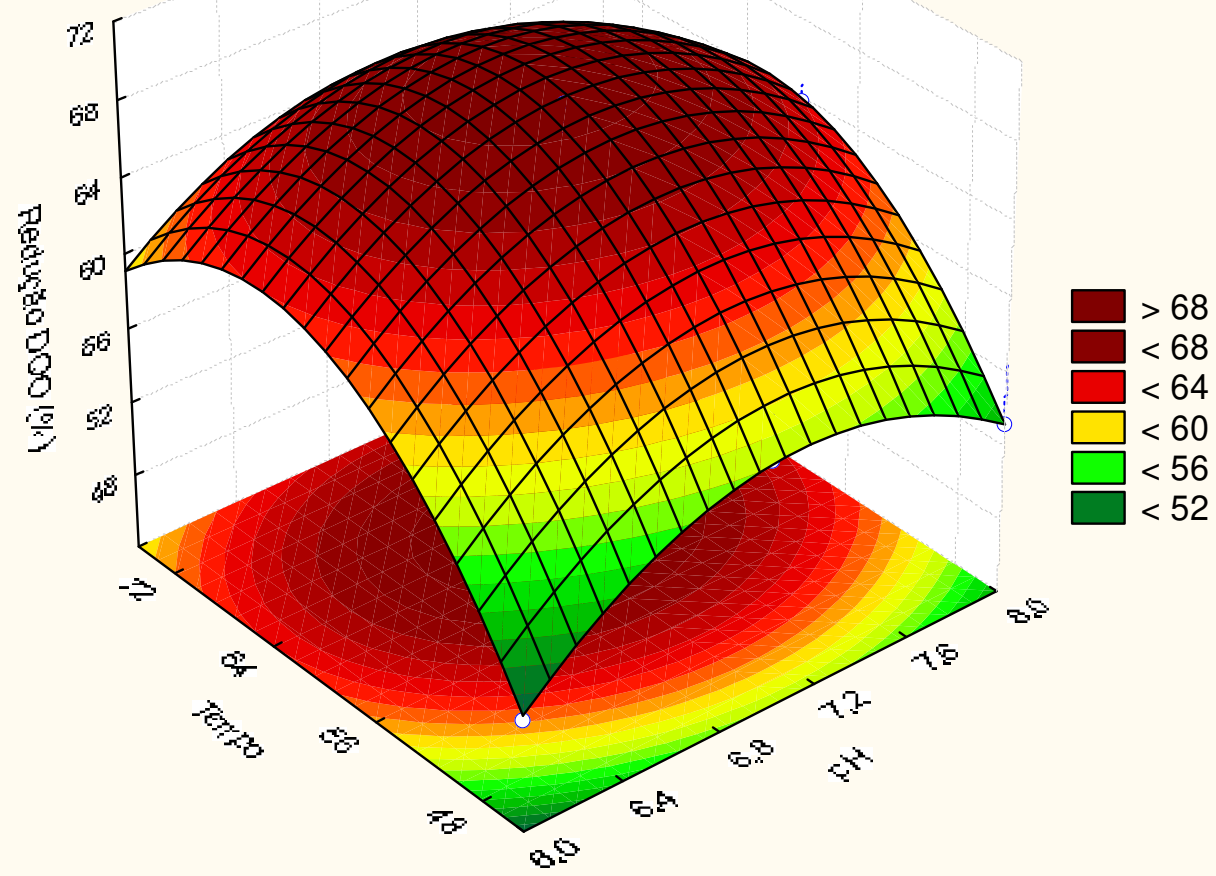

Fonte: Autoria própria (2020).

A superfície de resposta formada tem um formato de parábola invertida em razão de a eletrólise começar a perder o efeito devido à alta condutividade em tempos maiores que 60 minutos, assim a matéria flotada começa a se agregar novamente com todo o efluente. Verificou-se por intermédio do gráfico de superfície resposta para DQO que a porcentagem de remoção foi fortemente influenciada por ambos fatores, no qual o valor com melhor desempenho foi para pH 7,2 e tempo de eletrólise de 60 min. Assim, como a variável resposta anterior, os melhores resultados foram obtidos nos ensaios que permeiam o ponto central. 


\section{GESTÃO \& SUSTENTABILIDADE} AMBIENTAL

\section{¿UISUl}

Combatt et al. (2017) demonstram resultados da eletrocoagulação em efluentes de abatedouros de aves com valores de tempo de 2 min, $\mathrm{pH} 7,5$ e corrente de $15 \mathrm{~A}$ obtendo remoção superior a $86 \%$ de DQO, resultados mais expressivos que os obtidos neste estudo por conta da adição de compostos clorados inorgânicos e orgânicos no processo. Apesar da maior redução encontrada, o processo tem a desvantagem de um custo mais elevado.

De acordo com Borba et al. (2010) a adição de sal ou outro composto para controle da condutividade poderia alterar as superfícies de resposta apresentando valores e formatos diferentes, tornando-se uma superfície linear na relação redução da DQO em função do tempo de eletrólise.

O Gráfico de Pareto para os resultados de variação da condutividade elétrica pelo processo de eletrocoagulação (Figura 5) mostra que houve significância no aumento da condutividade elétrica apenas no termo de tempo linear.

Figura 5 - Gráfico de Pareto: Análise de variação da condutividade.

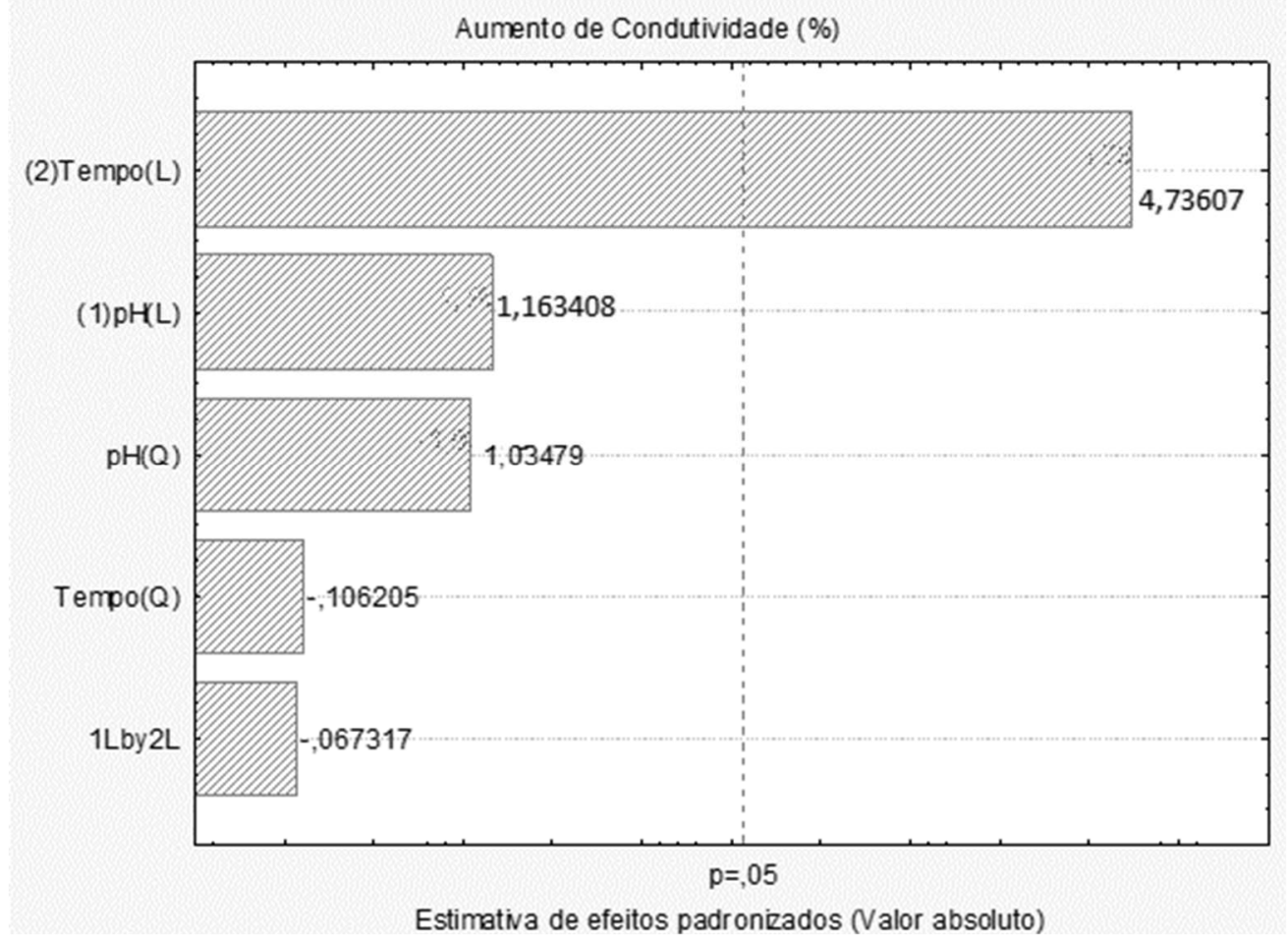

Fonte: Autoria própria (2020).

Como não houve nenhum tipo de adição de sais no processo a corrente elétrica iniciou com 5,5 A sendo que no tempo de 45 min apresentou-se com 2,02 A, no tempo de 60 min com 1,43 A e no tempo de 75 min com 0,67 A, ou seja, uma diminuição linear da corrente elétrica em função do tempo. 


\section{GESTÃO \& SUSTENTABILIDADE AMBIENTAL}

\section{sunisul simes}

Observa-se através da análise de variância (Tabela 7) que o modelo da ANOVA gerado a partir dos dados experimentais é valido para os intervalos de $\mathrm{pH}$ e tempo de eletrólise testados, sendo significativo para tempo linear.

Tabela 7 - Resumo ANOVA: Análise de DQO.

\begin{tabular}{c|c|c|c}
\hline & F calculado & -valor & Efeitos \\
\hline Tempo (linear) & 26,61114 & 0,000596 & 19,94599 \\
\hline Modelo Matemático & \multicolumn{3}{|c}{$Z=-5,59+0,66 y$} \\
\hline
\end{tabular}

Onde: $\mathrm{Z}=$ Aumento da condutividade (\%); $y=$ tempo de eletrólise; $\mathrm{p}$-valor inferiores a 0,05 indicam que o modelo explica a variação na resposta com significância estatística ao nível de $95 \%$ de probabilidade.

Fonte: Autores (2020).

$\mathrm{Na}$ Figura 6 pode-se observar o comportamento da condutividade elétrica em função do tempo de eletrólise para os limites estudados. A função resposta para o aumento da condutividade elétrica apresentou um comportamento diferente das funções resposta de turbidez e DQO, sendo linear em função do tempo de eletrólise.

Figura 6. Superfície resposta para variação de condutividade (\%).

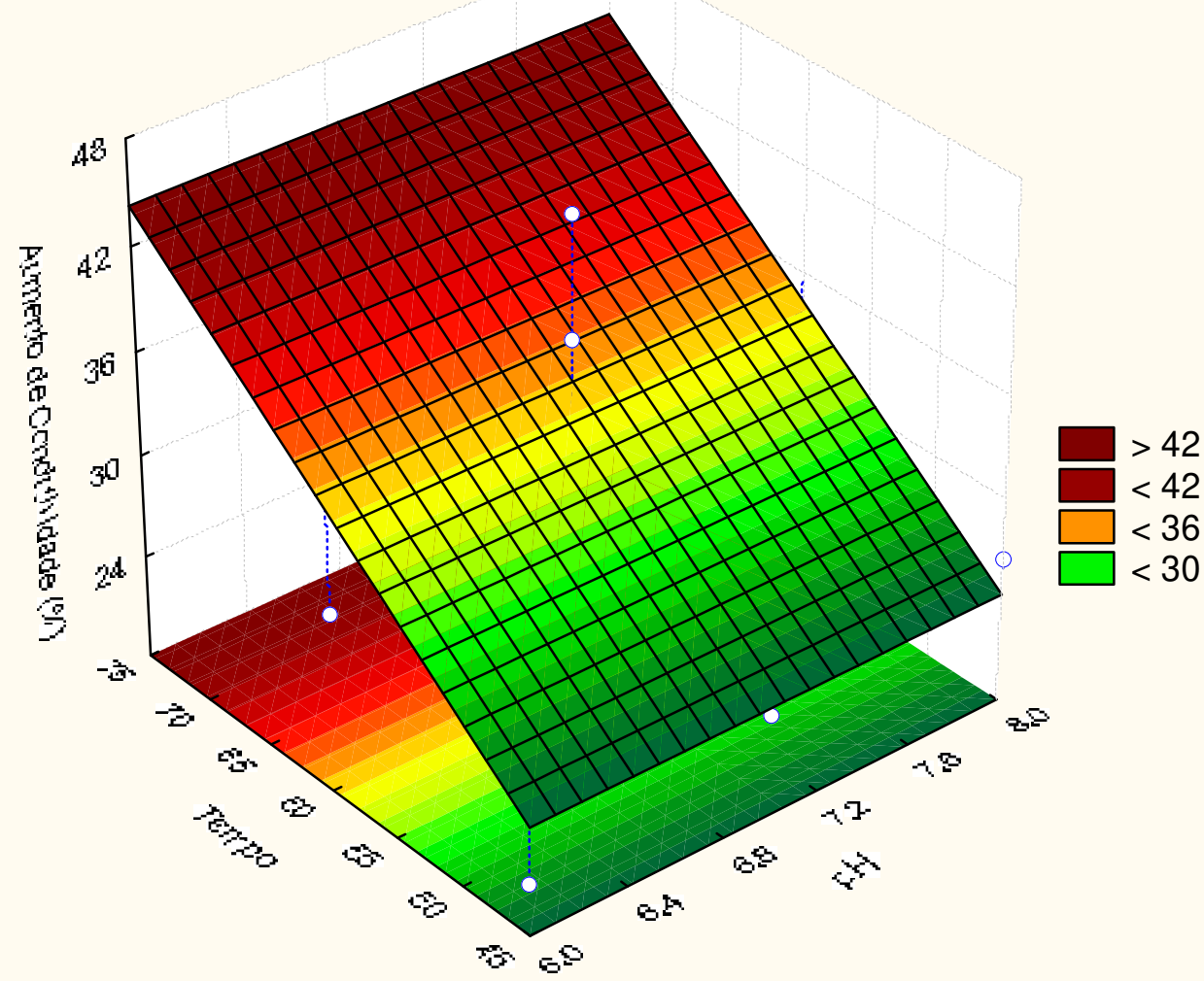

Fonte: Autores (2020). 


\section{GESTÃO \& SUSTENTABILIDADE} AMBIENTAL

\section{Unisul sumes}

Esta figura demostra que no tempo de 75 min, obteve-se a maior condutividade elétrica. Conforme o tempo de eletrólise avança, a resistência diminui, resultando em um aumento da condutividade, porém a eletrocoagulação teve uma tendência a zerar. Caso houvesse a adição de $\mathrm{NaCl}$ a variação de condutividade em relação ao tempo não existiria (DANESHVAR et al., 2007). O efluente antes e após o tratamento por eletrocoagulação é apresentado na Figura 7.

Figura 7- Efluente antes e após tratamento por eletrocoagulação.

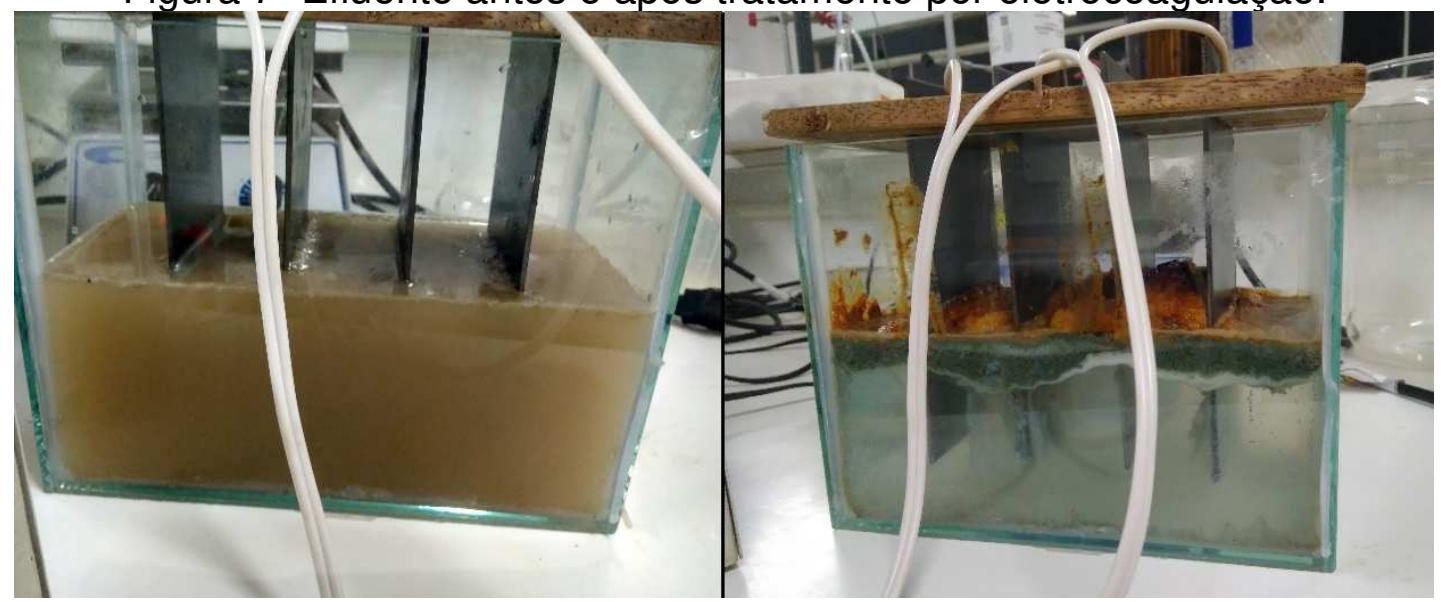

Fonte: Autores (2020).

A eletrocoagulação sem adição de sais aditivos forneceu resultados de remoção de turbidez, remoção de DQO e aumento de condutividade de $78,3 \%$, $68,8 \%$ e $46,0 \%$, respectivamente. Estes resultados foram relevantes ao presente estudo, porém insuficientes para serem usados como único tratamento de efluente. $A$ principal vantagem da não adição de $\mathrm{NaCl}$ ou qualquer outro composto para se manter baixa a condutividade é o fato de não se ter uma grande concentração de sólidos solúveis no efluente tratado. Outro aspecto importante é que com alta condutividade a potência dissipada pelo eletrocoagulador é baixa, causando um baixo consumo de energia.

\section{CONCLUSÕES}

O desenvolvimento do presente estudo possibilitou uma análise do processo de eletrocoagulação em um reator batelada, sem o uso de sais ou outros agentes para manter a condutividade do efluente bruto de um abatedouro de aves.

A partir dos ensaios obteve-se como melhores resultados a remoção de turbidez e de DQO de 78,3\%, 68,8 \% respectivamente, mostrando a eficiência do tratamento. As maiores eficiências encontradas foram nas condições próximas a 60 


\section{GESTÃO \& SUSTENTABILIDADE AMBIENTAL}

\section{unisul 5}

min e $\mathrm{pH} 7$, mostrando que a turbidez e a DQO têm comportamentos semelhantes frente a variação de tempo e $\mathrm{pH}$.

No processo de eletrocoagulação a condutividade está relacionada à remoção de DQO e turbidez, sendo que condutividades muito baixas ou muito elevadas resultam em uma menor remoção, sendo necessário seu controle.

Em abatedouros de aves a eletrocoagulação sem utilização de elementos que controlem a condutividade pode ser utilizada em batelada como tratamento secundário, principalmente por ser um tratamento rápido.

\section{REFERÊNCIAS}

ABREU, J. D.; ADA, C.; COSTA, A. F.; MARIAN, S.; KOSLOWSKI, L. A. D. Tratamento de efluente proveniente de serigrafia via eletrocoagulação. Brazilian Journal of Animal and Environmental Research. v.2, n.1, 2019.

AN, C.; HUANG, G.; YAO, Y.; ZHAO, S. Emerging usage of electrocoagulation technology for oil removal from wastewater: A review. Science of The Total Environment. v.1, p.537-556, 2017.

APHA. Standard methods for the examination of water and wastewater, 22nd ed.: American Public Health Association. American Water Works Association, Water Environment Federation. Washington, DC. 2012.

ASSOCIAÇÃO BRASILEIRA DE NORMAS TÉCNICAS. NBR 9898:1987: Preservação e técnicas de amostragem de efluentes líquidos e corpos receptores. Rio de Janeiro, 1987.

BAS, D.; BOYACI. J. Food Eng. p. 836, 2007.

BORBA, F. H.; MANENTI, D.R.; MÓDENES, A. N.; MORA, N. D.; ESPINOZAQUINÕNES, F. R.; PALÁCIO, S. M.; YASSUE, P. H.; NASCIMENTO, R. Avaliação da eficiência da técnica de eletrofloculação no tratamento de efluentes de indústrias de subprodutos avícolas. Estudos Tecnológicos. v.6, n.1, p.36-47, 2010.

CHEN, G. Electrochemical Technologies in wastewater treatment. Separation and Purification Technology. v.38, n.1, p11-41, 2004.

COMBATT, M. P. M.; MENDONÇA, R. C. S.; VALENTE, G. F. S.; SILVA, C. M. Validação do processo de eletrocoagulação e avaliação da eletrodissolução de eletrodos no tratamento de efluentes de abatedouros de aves. Química Nova. v.40, n.4, p.447-453, 2017.

DANESHVAR, N.; KHATAEE, A.; AMANI, G.; RASOULIFARD, M. H. Decolorization of C.I.Acid Yellow 23 solution by electrocoagulation process: Investigation of operational parameters anda evaluation of specific electrical energy consumption (SEEC). Journal of Hazardous Materials. v. 148, n.3, p. 566-572, 2007.

EMBRAPA. Estatística Brasil - Frangos de corte. 2019. Acessado em 27/03/2020. Page <https://www.embrapa.br/suinos-e-aves/cias/estatisticas/frangos/brasil>.

FERREIRA, A.; KUHN, S. S.; CREMONEZ, P. A.; DIETER, J.; TELEKEN, J. G.; SAMPAIO, S. C.; KUHN, P. D. Brazilian poultry activity waste: Destinations and 
GESTÃO \& SUSTENTABILIDADE AMBIENTAL

\section{¿Unisul}

energetic potential. Renewable and Sustainable Energy Reviews. v.81, n.2, p.30813089, 2018.

HAKIZIMANA, J. N.; GOURICH, B.; CHAFI, M.; STIRIBA, Y.; VIAL, C.; DROGUI, P.; NAJA, J. Electrocoagulation process in water treatment: A review of electrocoagulation modeling approaches. Desalination. v.404, p.1-21, 2017.

INSTITUTO AMBIENTAL DO PARANÁ. Parâmetros de lançamentos de efluentes líquidos. 2020. Acessado em 10/04/2020. Page <http://www.iap.pr.gov.br/pagina49.html>.

KRUG, T. C.; HERMES, E. Management of liquid effluents and proposal of a new treatment system in poultry slaughterhouse of the west region of Paraná. Brazilian Journal of Animal and Environmental Research. v.2, n.1, p.593-600, 2019.

KUMAR, A.; NIDHEESH P. V.; KUMAR, M. S. Composite wastewater treatment by aerated electrocoagulation and modified peroxi-coagulation processes. Chemosphere. v.205, p.587-593, 2018.

KUMAR, M.; PONSELVAN, I.A.; MALVIYA, J.R.; SRIVASTAVA, V.C.; MALL, I.D. Treatment of bio-digester efluent by electrocoagulation using iron electrodes. Journal of Hazardous Materials. v. 165, n. 1-3, p. 345-352, 2009.

LIMA, G. V.; ALVES, P. A. S. Análise comparativa para implementação de eletrólise para tratamento de águas residuárias na região metropolitana de Goiânia. 2018.

MÓdENES, A. N.; ESPINOZA-QUINONEZ, F. R.; YASSUE, P. H.; PORTO, T. M.; THEODORO, P. S. Aplicação da técnica de eletrocoagulação no tratamento de efluentes de abatedouro de aves. Engenharia Sanitária Ambiental. v.22, n.3, p.571578, 2017.

MOUSSA, D. T.; EL-NAAS, M. H.; NASSER, M.; AL-MARRI, M. J. A Comprehensive review of electrocoagulation for water treatment: Potentials and challenges. Journal of Environmental Management. v.186 part 1, p.24-41, 2017.

ROSHINI, P. S.; GANDHIMATHI, R.; RAMESH, S. T.; NIDHEESH, P. V. Combined electro-fenton and biological processes for the treatment of industrial textile effluent: mineralization and toxicity analysis. J. Hazardous, Toxic, Radioact. Waste. v.21, 2017.

SANTOS, G. O. S.; PUPO, M. M. S.; VASCONCELOS, V. M.; EGUILUZ, K. I. B.; BANDA, G. R. S. Electroflotation. Electrochemical Water and Wastewater Treatment. P.77-118, 2018.

SILVA, A. P.; ARGONDIZO, A.; SEKI, C. C. Utilização de eletrodo de leito fixo no tratamento de efluente textile por eletrocoagulação. Brazilian Journal of Development. v.6, n.3, 2020.

SOUZA, P. C.; PEREIRA, N. C.; GONÇALVES, M. S.; CANSOLIN FILHO, N.; RODRIGUES, P. H.; JAMARIM, V. M. Estudo do Tratamento de efluente têxtil através de processos de coagulação/floculação e eletrocoagulação. Revista Exacta. v.9, n.2, 2016.

SRIDHAR, R.; SIVAKUMAR, V.; PRINCE IMMANUEL, V.; PRAKASH MARAN, J. Development of model for treatment of pulp and paper industry bleaching effluent using 


\section{GESTÃO \& SUSTENTABILIDADE}

AMBIENTAL

\section{¿Unisul sines}

response surface methodology. Environ Prog Sustainable Energy. v.31 p.558-565. 2012.

THIRUGNANASAMBANDHAM, K.; SIVAKUMAR, V.; PRAKASH MARAN, J. Efficiency of electrocoagulation method to treat chicken processing industry wastewater-modeling and optimization. Journal of the Taiwan Institute of Chemical Engineers. v.45, n.5, p.2427-2435. 2014.

YAVUZ, Y; OGUTVEREN, U. B. Treatment of industrial estate wastewater by the application of electrocoagulation process using iron electrodes. Journal of Environmental Management. v.207, p.151-158, 2018. 